\title{
Propiedades psicométricas de la Escala Hospitalaria de Ansiedad y Depresión (HADS) en una población de pacientes oncológicos mexicanos
}

\author{
Oscar Galindo Vázquez, ${ }^{1,2}$ Corina Benjet, ${ }^{3}$ Francisco Juárez García, ${ }^{3}$ Edith Rojas Castillo, ${ }^{2}$ Angélica Riveros \\ Rosas, ${ }^{4}$ José Luis Aguilar Ponce, ${ }^{5}$ Miguel Ángel Álvarez Avitia, ${ }^{6}$ Salvador Alvarado Aguilar ${ }^{2}$
}

\section{Artículo original}

\section{ABSTRACT}

\section{Background}

Symptoms of anxiety and depression are among the major mental health problems in cancer patients. These symptoms affect the quality of life and treatment adherence, and are associated with other symptoms and longer hospital stays. Valid and reliable screening instruments such as the Hospital Anxiety and Depression Scale (HADS), have made possible the detection of possible cases of depression and anxiety in medically ill patients. However, the psychometric properties of this instrument have not been documented in different types of cancer diagnoses in the Mexican population.

\section{Objective}

To determine the psychometric properties of the HADS in a sample of patients with cancer from the Mexican population.

\section{Method}

Four hundred patients from the National Cancer Institute participated, of which 226 were women $(56.5 \%)$ and 174 men $(43.6 \%)$, with a mean age of $47.4 \pm 14.1$ years. Participants completed concurrently the HADS as well as the following inventories: 1. Beck Depression, 2. Beck Anxiety and 3. Distress Thermometer.

\section{Results}

A factor analysis adjusted to two factors explained $48.04 \%$ of the variance, with 12 items loading on these two factors in a way similar to the original version. The internal consistency of the overall scale was satisfactory $(\alpha=0.86)$. Cronbach's alphas for each subscale were .79 and .80 . The concurrent validity assessed by way of correlations with concurrent measures showed significant associations (Pearson $r=51-71, p<0.05)$.

\section{Discussion and conclusion}

The HADS has adequate construct validity, internal consistency and concurrent validity for its use in cancer patients from the Mexican population. The relevance of these results is a cost effective tool to provide timely mental health care early in oncological treatment for those in need. Detecting anxiety and depression symptoms through the HADS may benefit cancer patients by ensuring appropriate care that may increase their quality of life and treatment adherence, and reduce their hospital stays.

Key words: Anxiety, cancer, depression, patients, Mexican population.

\section{RESUMEN}

\section{Antecedentes}

La sintomatología ansiosa y depresiva es parte de los principales problemas de salud mental en pacientes oncológicos, lo cual afecta la calidad de vida y la adhesión al tratamiento, además de que se asocia con mayor número de síntomas y estancia hospitalaria. Mediante instrumentos de tamizaje válidos y confiables, como la Escala hospitalaria de ansiedad y depresión (HADS), ha sido posible detectar posibles casos en pacientes hospitalarios. Sin embargo, hasta ahora no se habían caracterizado las propiedades psicométricas en pacientes oncológicos en población mexicana.

\section{Objetivo}

Determinar las propiedades psicométricas de la HADS en una muestra de pacientes oncológicos.

\section{Método}

Participaron 400 pacientes del Instituto Nacional de Cancerolo gía, de los cuales 226 eran mujeres (56.5\%) y 174 eran hombres $(43.6 \%)$; la edad promedio fue de $47.4 \pm 14.1$ años. Los participantes contestaron, además de la HADS, los siguientes inventarios: depresión de Beck, ansiedad de Beck, termómetro de distrés.

\section{Resultados}

Un análisis factorial ajustado a dos factores presentó un instrumento con 12 reactivos, similar a la versión original. La consistencia interna

\footnotetext{
Facultad de Psicología, Universidad Nacional Autónoma de México.

Servicio de Psico-oncología, Instituto Nacional de Cancerología.

Dirección de Investigaciones Epidemiológicas y Psicosociales, Instituto Nacional de Psiquiatría Ramón de la Fuente Muñiz.

División de Investigación, Facultad de Contaduría y Administración, Universidad Nacional Autónoma de México.

Subdirección de Medicina Interna, Instituto Nacional de Cancerología.

Servicio Oncología Médica, Instituto Nacional de Cancerología.
}

Correspondencia: Dr. Oscar Galindo Vázquez. Servicio de Psico-oncología, Instituto Nacional de Cancerología. Av. San Fernando 22. Sección XVI, Tlalpan, 14080, México, DF. Tel: 5628-0400 Ext. 41 E-mail: psigalindo@yahoo.com.mx

Recibido primera versión: 22 de septiembre de 2013. Segunda versión: 9 de julio de 2014. Tercera versión: 14 de septiembre de 2014. Aceptado: 22 de octubre de 2014. 
de la escala global mostró un índice satisfactorio $(\alpha=0.86)$. Los alfas de Cronbach de cada subescala tuvieron un valor de .79 y .80 que explicaron el $48.04 \%$ de la varianza. La validez, por medio de correlación con las medidas concurrentes, mostró resultados significativos ( $r$ de Pearson de .51 a .71, $p<0.05$ ).

\section{Discusión y conclusión}

La HADS en pacientes con cáncer en población mexicana presentó adecuadas características psicométricas. La relevancia de los resul- tados obtenidos radica en que se trata de una población que puede llegar a requerir atención oportuna en salud mental en etapas tempranas de su tratamiento. La detección de sintomatología ansiosa y depresiva por medio de la HADS deriva en beneficios para la población oncológica y en estrategias funcionales de atención adecuada y costo-efectivas.

Palabras clave: Ansiedad, cáncer, depresión, pacientes, población mexicana.

\section{ANTECEDENTES}

El cáncer se ubica como uno de los principales problemas de salud pública en el mundo. En este sentido, es necesaria la identificación y atención de problemas psicológicos en pacientes que presentan estas características. ${ }^{1}$

Durante el proceso de la enfermedad oncológica, el diagnóstico, tratamiento y periodo de seguimiento, los síntomas de ansiedad y depresión constituyen una constante en la vida del paciente con cáncer., ${ }^{2,3}$

Diversas investigaciones a nivel mundial muestran que, en la población oncológica, del 15 al 58\% de pacientes presentan sintomatología depresiva, y de 24 a 66\%, sintomatología ansiosa, los cuales representan índices superiores a los reportados entre la población general. - $^{4}$

Es fundamental el diagnóstico y tratamiento de ambas patologías psicológicas, ya que pueden influir negativamente en la calidad de vida, duración del ingreso hospitalario, ideación suicida, autocuidado, adhesión al tratamiento médico y síntomas como dolor, nauseas, vómito y fatiga. ${ }^{9-12}$

Una de las herramientas más prácticas y económicas para la detección oportuna de sintomatología ansiosa y depresiva la constituyen los instrumentos de tamizaje válidos y confiables, ya que proporcionan una aproximación para la detección de posibles casos clínicos. Con el fin de identificar la presencia de sintomatología ansiosa y depresiva en el contexto hospitalario, la Escala hospitalaria de ansiedad y depresión (HADS por sus siglas en inglés), desarrollada por Zigmond y Snaith (1983), ${ }^{13}$ es una herramienta autoaplicada conformada por 14 ítems que permite utilizarse en medios hospitalarios no psiquiátrico o en atención primaria. Es un instrumento que considera dimensiones cognitivas y afectivas, que omite aspectos somáticos ${ }^{14}$ (insomnio, fatiga, pérdida de apetito, etc.) y así evita atribuirlos a la enfermedad. La escala original ha sido adaptada y validada en diversas poblaciones y culturas, y ha mostrado siempre una sensibilidad y especificidad adecuada en la discriminación de ansiedad y depresión. ${ }^{15-18}$

Bjelland et al. (2002), en una revisión de 747 estudios sobre la validez de la HADS en diferentes enfermedades crónico degenerativas, encontraron una adecuada estructura factorial del instrumento con un alfa de Cronbach para el factor de sintomatología ansiosa de 0.68 a 0.93 y para sinto- matología depresiva de .67 a .90 . En relación con la sensibilidad y especificidad para ambos factores es de 0.80 . La conclusión es que la HADS es un instrumento válido y confiable para la población hospitalaria. ${ }^{19}$

En población oncológica ha mostrado ser un medio adecuado para identificar sintomatología ansiosa y depresiva, al arrojar rangos de sensibilidad de entre .74 y .84, especificidad de .78 a .80 , una alta consistencia interna (alfas entre .83 y .85$)$, alta confiabilidad test retest $(r=.75)$ y una adecuada validez convergente $(\mathrm{p}<.05)$, así como una estructura factorial semejante con la versión original. ${ }^{14,18,20,21}$ Adicionalmente, la HADS ha demostrado ser un instrumento adecuado y sensible a los cambios tanto durante el curso de la enfermedad, como en respuesta al tratamiento psicoterapéutico e intervención psicofarmacológica. ${ }^{19,21,22}$

Sin embargo, faltan datos sobre su validez y confiabilidad en población mexicana con cáncer. Por lo tanto, el objetivo de este estudio es obtener las propiedades psicométricas de la HADS. Como resultado de esta validación, se facilitará la identificación de sintomatología de ansiedad y depresión, la evaluación de efectos de intervenciones psicológicas, la comparación de hallazgos con estudios internacionales y el realizar recomendaciones que ayudarán a mejorar las opciones de atención psicológica de los pacientes con cáncer.

\section{MÉTODO}

\section{Participantes}

Se incluyeron 400 pacientes oncológicos de ambos sexos entre 16 y 80 años (cuadro 1). La obtención de la muestra fue por disponibilidad en el Instituto Nacional de Cancerología (INCAN).

En la HADS las puntuaciones medias fueron de 6.48 ( \pm 3.70$)$ para ansiedad y de 5.07( \pm 3.83 ) para depresión; el puntaje total promedio de la muestra fue de $11.34( \pm 6.50)$.

\section{Procedimiento}

La muestra de participantes se obtuvo durante las consultas de los Servicios de Oncología médica, Cirugía, Radioterapia y Psico-oncología, tanto en consulta externa como en hospi- 
Cuadro 1. Descripción de la muestra

\begin{tabular}{|c|c|c|c|c|c|}
\hline & $f$ & $\%$ & & $f$ & $\%$ \\
\hline \multirow[t]{2}{*}{$\mathrm{N}$} & 400 & 100 & Diagnóstico & & \\
\hline & & & Mama & 137 & 34.1 \\
\hline \multirow{2}{*}{$\begin{array}{l}\text { Edad (Rango) } \\
16-80\end{array}$} & & & Urogenitales & 71 & 18.7 \\
\hline & & & $\begin{array}{l}\text { Cabeza y } \\
\text { cuello }\end{array}$ & 46 & 11.5 \\
\hline Sexo & & & Ginecológicos & 35 & 8.6 \\
\hline Masculino & 174 & 43.6 & Pulmón & 31 & 7.5 \\
\hline \multirow[t]{2}{*}{ Femenino } & 226 & 56.5 & Hematológicos & 30 & 7.4 \\
\hline & & & Gástricos & 23 & 5.6 \\
\hline Escolaridad & & & $\begin{array}{l}\text { Neoplasias de } \\
\text { la piel }\end{array}$ & 21 & 5.4 \\
\hline Ninguno & 28 & 7.0 & Otros & 6 & 1.2 \\
\hline Primaria & 96 & 24.8 & & & \\
\hline Secundaria & 112 & 28.0 & Enfermedad & & \\
\hline Bachillerato & 91 & 22.8 & Localizada & 76 & 19.0 \\
\hline Licenciatura & 66 & 16.5 & Locorregional & 150 & 37.5 \\
\hline Posgrado & 7 & 1.8 & Metastásica & 174 & 43.5 \\
\hline Estado civil & & & Tratamiento & & \\
\hline Soltero(a) & 94 & 23.6 & QT & 150 & 37.5 \\
\hline $\begin{array}{l}\text { Casado o unión } \\
\text { libre }\end{array}$ & 217 & 54.3 & Cirugía + QT & 78 & 19.5 \\
\hline $\begin{array}{l}\text { Separado(a) o } \\
\text { divorciado(a) }\end{array}$ & 43 & 10.8 & $\begin{array}{l}\text { Cirugía + QT } \\
+ \text { RT }\end{array}$ & 58 & 14.5 \\
\hline Viudo & 21 & 5.3 & $\mathrm{QT}+\mathrm{RT}$ & 50 & 12.5 \\
\hline Otro & 25 & 6.3 & Cirugía & 25 & 6.3 \\
\hline & & & Otros & 39 & 9.7 \\
\hline
\end{tabular}

Nota: $Q T=$ Quimioterapia, $R T=$ Radioterapia.

talización durante el periodo comprendido entre junio y diciembre de 2012. El proyecto fue aprobado por los Comités Científico y de Bioética del INCAN y cada uno de los participantes aceptó participar en el presente estudio mediante la comprensión y firma del consentimiento informado de investigación.

Los participantes fueron seleccionados a partir de los siguientes criterios de inclusión: 1. Pacientes en tratamiento oncológico (curativo o paliativo) o periodo de seguimiento (máximo seis meses), 2. Cualquier tipo de diagnóstico oncológico y estadio del proceso oncológico, 3. Saber leer y escribir y 4. Índice Karnofsky igual o mayor a 60. Criterios de exclusión: 1. Pacientes que presenten cuadros psiquiátricos severos y 2. Con complicaciones vasculares mayores, disnea, deficiencias cognitivas así como problemas auditivos y visuales severos.

\section{Instrumentos}

Escala hospitalaria de ansiedad y depresión. La HADS consta de dos subescalas de siete ítems intercalados. La subescala de depresión está centrada en el concepto de anhedonia como síntoma nuclear de este cuadro clínico y que diferencia primordialmente la ansiedad de la depresión. Cada reactivo tiene cuatro posibles respuestas que puntúan de 0 a 3, para un total de 0 a 21. Tanto para las puntuaciones de ansiedad como de depresión se considera normal de cero a siete, dudoso de ocho a diez y problema clínico a partir de once.

De acuerdo con Herrmann (1997), diversos estudios reportan tasas de respuesta del 95 al 100\%. La escala se puede contestar de cinco a seis minutos, lo que hace sencilla su aplicación por parte del equipo de salud.

En México, la HADS ha sido validada por Villegas $(2004)^{23}$ en mujeres en puerperio complicado, embarazo de alto riesgo, ginecología y oncología; por Rojas $(1991)^{24}$ en población geriátrica; por Whaley (1992) ${ }^{25}$ en pacientes quemados, y por López-Alveranga et al. $(2002)^{26}$ en pacientes obesos.

Inventario de depresión de Beck. El BDI (por sus siglas en inglés) (1961), ${ }^{27}$ fue estandarizado para población mexicana por Jurado, Villegas, Méndez, Rodríguez, Loperena y Varela (1998) ${ }^{28}$ y diseñado para evaluar la intensidad de la sintomatología depresiva. Es autoaplicable y consta de 21 reactivos, con cuatro opciones de respuesta que describen el espectro de severidad de la categoría sintomática y conductual. Las categorías evaluadas por el BDI son 1. ánimo, 2. pesimismo, 3. sensación de fracaso, 4. insatisfacción, 5. sentimientos de culpa, 6 . sensación de castigo, 7. autoaceptación, 8. autoacusación, 9. ideación suicida, 10. llanto, 11. irritabilidad, 12. aislamiento, 13. indecisión, 14. imagen corporal, 15. rendimiento laboral, 16. trastornos del sueño, 17. fatigabilidad, 18. apetito, 19. pérdida de peso, 20. preocupación somática y 21. pérdida de la libido. Las propiedades psicométricas del BDI en población mexicana muestran que la confiabilidad por consistencia interna obtenida fue alfa de Cronbach=.87. El análisis factorial mostró que se compone por tres factores. En relación con la validez concurrente entre el BDI y la Escala de Zung, ${ }^{29}$ la correlación estadística fue $\mathrm{r}=0.70, \mathrm{p}<0.00$.

Inventario de ansiedad de Beck. El BAI (por sus siglas en inglés) (Beck, Epstein, Brown y Steer, 1988) ${ }^{30}$ fue estandarizado para población mexicana por Robles, Varela, Jurado y Páez (2011) ${ }^{31}$ Es un instrumento de autorreporte y consta de 21 reactivos que determinan la severidad con que se presentan las categorías sintomáticas y conductuales. A continuación, se enlistan las categorías evaluadas por el BAI: 1. subjetivo, 2. neurofisiológico, 3. autonómico y 4. pánico. El BAI se caracteriza por una alta consistencia interna (alfas superiores a 0.90), validez divergente moderada (correlaciones menores a 0.60) y validez convergente adecuada (correlaciones mayores a 0.50). Los análisis factoriales han extraído cuatro factores principales, que se han denominado subjetivo, neurofisiológico, autonómico y pánico. Las propiedades psicométricas del BAI para población mexicana se caracterizan por una alta consistencia interna (alfas 0.84 y 0.83), con un alto coeficiente de confiabilidad test-retest $(r=.75)$, validez convergente y estructura factorial adecuada.

Termómetro de Distrés. El termómetro de Distrés (Holland, 1999) ${ }^{32}$ fue validado para población mexicana por 
Almanza-Muñoz, Juárez y Pérez (2008), ${ }^{33}$ y consta de dos partes. La primera es una escala análoga visual, que va de 0 a 10, para evaluar el distrés experimentado en la última semana. La segunda plantea una lista de 35 problemas prácticos comunes en pacientes con cáncer. La validación convergente por medio del análisis ROC mostró área bajo la curva de 0.63 , sensibilidad de $93 \%$, especificidad de $76 \%$, valor predictivo positivo de $82 \%$ y valor predictivo negativo de $90 \%$, con un punto de corte de cuatro o más, lo cual arroja una prevalencia del distrés de $61.8 \%$.

\section{Análisis estadísticos}

Se determinó la estructura factorial por medio de un análisis factorial de componentes principales con rotación Varimax. Los criterios utilizados para el análisis factorial fueron: 1 . cargas factoriales $\geq .40 ; 2$. factores con por lo menos tres reactivos y 3 . coeficiente de consistencia interna por cada factor alfa de Cronbach $\geq .60$. La relación entre la HADS y las medidas concurrentes se analizó mediante el coeficiente de correlación de Pearson y la consistencia interna con el alfa de Cronbach. Se establecieron los puntos de corte de ambas subescalas, ajustándose al percentil 75 como punto de corte que plantea una diferencia estadística y clínica entre los grupos con menor y mayor cantidad de atributo $(<0.05)$.

\section{RESULTADOS}

\section{Puntos de corte}

Para calcular los puntos de corte se tomaron los valores obtenidos a partir del percentil 75 para considerar como caso leve de sintomatología ansiosa o depresiva.
Igualmente, para considerar pacientes sin depresión (05), depresión leve (6-8), depresión moderada (9-11) y depresión grave (12 en adelante). Para la subescala de ansiedad: sin ansiedad (0-5), ansiedad leve (6-8), ansiedad moderada (9-11) y ansiedad grave (12 en adelante).

\section{Estructura factorial}

Se obtuvo en la prueba de esfericidad de Bartlett una $(\mathrm{p}=.001)$ y un índice de adecuación muestral $(\mathrm{KMO}=0.83)$.

El primer análisis factorial exploratorio (método de componentes principales y rotación Varimax) con 14 reactivos arrojó un modelo con tres factores con autovalores arriba de 1.00; aunque explicaba el $53.74 \%$ de la varianza, no concordaba con los dos factores de la versión original. En el tercer factor se agruparon los reactivos 7, "Puedo estar sentado(a) tranquilamente y sentirme relajado(a)", y 8, "Me siento como si cada vez estuviera más lento", por lo que fueron eliminados debido a que no correspondieron conceptualmente con las subescalas originales. Por lo que se realizó un análisis factorial confirmatorio con 12 reactivos que obtuvo el $48.04 \%$ de la varianza (cuadro 2). Adicionalmente se realizaron dos análisis factoriales con los 12 reactivos. En ambas submuestras (pacientes de consulta externa y hospitalización); se obtuvieron el $46.73 \%$ y el $48.42 \%$ de la varianza, respectivamente.

\section{Consistencia interna}

El alfa de Cronbach de la subescala de ansiedad de la HADS (6 reactivos) fue de 0.79 , en tanto que la subescala de depresión (6 reactivos), de 0.80 . El alfa de Cronbach, con 12 reactivos, alcanzó 0.86 . El alfa de Cronbach en ambas submuestras (consulta externa y hospitalización) fue de 0.82 y 0.87 . En términos generales, la consistencia puede considerarse adecuada.

Cuadro 2. Análisis factorial de elementos

\begin{tabular}{|c|c|c|c|}
\hline Escala global $\alpha=.86$ Varianza explicada $=48.04 \%$ & $\begin{array}{c}\text { Carga } \\
\text { factorial }\end{array}$ & $\begin{array}{l}\text { Varianza } \\
\text { explicada }\end{array}$ & $\begin{array}{c}\text { Alfa de } \\
\text { Cronbach }\end{array}$ \\
\hline HADS - A Subescala ansiedad & & $36.55 \%$ & $\alpha=.79$ \\
\hline 1. Me siento tenso(a) o nervioso(a) & .689 & & \\
\hline 3. Tengo una sensación de miedo, como si algo horrible me fuera a suceder & .793 & & \\
\hline 5. Tengo mi mente llena de preocupaciones & 628 & & \\
\hline 9. Tengo una sensación extraña, como de "aleteo" en el estómago & .519 & & \\
\hline 11. Me siento inquieto(a), como si no pudiera parar de moverme & .641 & & \\
\hline 13. Me asaltan sentimientos repentinos de pánico & .736 & & \\
\hline HADS - D Subescala depresión & & $11.49 \%$ & $\alpha=.80$ \\
\hline 2. Todavía disfruto con lo que antes me gustaba & .658 & & \\
\hline 4. Puedo reírme y ver el lado divertido de las cosas & .756 & & \\
\hline 6. Me siento alegre & .650 & & \\
\hline 10. He perdido el interés en mi aspecto personal & .649 & & \\
\hline 12. Me siento optimista respecto al futuro & .663 & & \\
\hline 14. Me divierto con un buen libro, la radio o un programa de televisión & .738 & & \\
\hline
\end{tabular}


Cuadro 3. Correlaciones entre la HADS y medidas concurrentes

\begin{tabular}{lccccc}
\hline & 1 & 2 & 3 & 4 & 5 \\
\hline 1. HADS total & & & & & \\
2. HADS - A & $.849^{*}$ & & & & \\
3. HADS - D & $.911^{*}$ & $.557^{*}$ & & & \\
4. BDI & $.711^{*}$ & $.560^{*}$ & $.682^{*}$ & & \\
5. BAl & $.612^{*}$ & $.537^{*}$ & $.545^{*}$ & $.660^{*}$ & \\
6. Distrés & $.532^{*}$ & $.505^{*}$ & $.443^{*}$ & $.477^{*}$ & $.530^{*}$ \\
\hline
\end{tabular}

* Correlaciones Pearson con $p<.001$

\section{Validez externa por medio de correlaciones con medidas concurrentes}

El cuadro 3 muestra el resumen de las asociaciones entre la HADS con las medidas concurrentes administradas BDI, BAI y Termómetro de distrés. Todas las correlaciones fueron positivas y estadísticamente significativas ( $\mathrm{r}$ de Pearson de 0.51 a $0.71, \mathrm{p}<0.05$ ) (cuadro 3). Adicionalmente se correlacionó la HADS con el índice Karnofsky ( $\mathrm{r}=-.337)$, HAD-A $(\mathrm{r}=-.244)$ y la HAD-D ( $\mathrm{r}=-.238) \mathrm{p}<0.01$, lo cual indica una correlación inversamente proporcional.

\section{DISCUSIÓN Y CONCLUSIÓN}

La evaluación de la sintomatología ansiosa y depresiva en pacientes oncológicos es de gran relevancia debido a su relación con afectaciones severas durante el tratamiento. La HADS de Zigmond y Snaith (1983) ${ }^{13}$ es un instrumento diseñado para medir estos constructos, el cual ha mostrado adecuadas características psicométricas replicadas en estudios en diversos países. La HADS en población mexicana presentó una estructura similar y confiabilidad aceptable. Sin embargo, la omisión de los ítems 7 y 8 pueden indicar un constructo diferente. A pesar de que la escala pretende excluir síntomas somáticos, estos dos ítems parecen no cumplir con el objetivo. Los hallazgos pueden indicar un componente adicional, como el identificado por Rico et al., 2005, ${ }^{20}$ con los reactivos 8 y 9 y los estudios identificados por Bjelland et al., 2002.19

Una validez concurrente adecuada, mediante asociaciones significativas con el BDI y el BAI, que incluyen elementos somáticos, así como el distrés, que es un malestar emocional que interfiere con la habilidad para enfrentar el cáncer, indican que la HADS plantea un constructo centrado en la anhedonia con elementos cognitivos conductuales estables. Finalmente, asociaciones inversamente proporcionales con el Índice Karnofsky confirman que a mayores síntomas de ansiedad y depresión, el nivel de funcionalidad del paciente disminuye. En relación con los puntos de corte, difieren del artículo original de Zigmond y Snaith (1983), ${ }^{13}$ debido a que se modificó el número de reactivos para cada subescala.

La ventaja de la HADS para esta población oncológica sobre otros instrumentos que evalúan sintomatología ansiosa y depresiva, es que no incluye síntomas somáticos que pueden explicarse por el cáncer y su tratamiento. Esto puede contribuir a una sobreestimación en los demás instrumentos.

Al ser un instrumento breve, fácil de aplicar y confiable para la práctica clínica y la investigación en población oncológica, resulta relevante para determinar la magnitud de la problemática, prevención e implementación de acciones para el tratamiento.

Un adecuado reconocimiento de las afectaciones psicosociales es crucial para identificar al grupo de pacientes con afectaciones psicológicas, realizar una oportuna derivación a los servicios de salud mental ${ }^{34,35}$ y proveer las intervenciones psicológicas que han mostrado efectos benéficos en este grupo de pacientes ${ }^{36,37}$ con el objetivo de mantener parámetros aceptables de bienestar psicológico en esta población.

Entre las limitaciones de este trabajo se encuentra la falta de una entrevista psiquiátrica para la confirmación de la sintomatología ansiosa y depresiva.

Finalmente, los datos apoyan la necesidad de incrementar los esfuerzos orientados a volver más accesibles los servicios de salud mental en este grupo de pacientes.

\section{Financiamiento}

Ninguno.

\section{Conflicto de intereses}

Los autores declararon no tener conflicto de intereses.

\section{REFERENCIAS}

1. Zabora J, Brintzenhofeszoc K, Curbow B, Hooker C et al. Piantadosi S. The prevalence of psychological distress by cancer site. Psycho-Oncology 2001;10:19-28.

2. Spiegel D. Cancer and depression. British J Psychiatry 1996;168(30):109116.

3. Maté J, Hollenstein M, Gil F. Insomnio, ansiedad y depresión en el paciente oncológico. Psicooncología 2004;1(2):211-230.

4. Derogatis LR, Morrow GR, Fetting J. The prevalence of psychiatric disorders among cancer patients. JAMA 1983:249:751-757.

5. Jadoon NA, Munir W, Shahzad MA, Choudhry ZS. Assessment of depression and anxiety in adult cancer outpatients: a cross-sectional study. BMC Cancer 2010;10(594):1471-2407.

6. Krauss HA, Hauss JP, Höckel M, Kortmann RD et al. Anxiety and depression in cancer patients compared with the general population. European J Cancer Care 2010;19:522-529.

7. Massie MJ. Prevalence of depression in patients with cancer. J National Cancer Institute Monogr 2004;32:57-71.

8. Henriksson MM, Isometsä ET, Hietanen PS, Aro HM et al. Mental disorders in cancer suicides. J Affect Disord 1995;36:11-20.

9. Holland JC. Psycho-oncology. Nueva York: Oxford University Press; 1998.

10. DiMatteo MR, Lepper HS, Croghan TW. Depression is a risk factor for noncompliance with medical treatment meta-analysis of the effects of anxiety and depression on patient adherence. Arch Intern Med 2000;160:2101-2107.

11. Vahdaninia M, Omidvari S, Montazeri A. What do predict anxiety and depression in breast cancer patients? A follow-up study. Soc Psychiat Epidemiol 2010;45:355-361. 
12. Bottomley A. Depression in cancer patients: a literature review. Eur J Cancer 1998;7:181-191.

13. Zigmond AS, Snaith RP. The hospital anxiety and depression Scale. Acta psychiatric Scandinavic 1983;67:361-370.

14. Costantini M, Musso M, Viterbori P, Bonci F et al. Detecting psychological distress in cancer patients: validity of the Italian version of the hospital anxiety and depression Scale. Support-Care-Cancer 1999;7(3):121-127.

15. Lisspers J, Nygren A, Soderman E. Hospital anxiety and depression Scale (HAD): some psychometric data for a Swedish sample. Acta Psychiatr Scand 1997;96:281-286.

16. Herrmann C. International experiences with the hospital anxiety and depression scale a review of validation data and clinical results. J Psychosomatic Research 1997;42(1):17-41.

17. Carroll BT, Kathol RG, Noyes A. Screening for depression and anxiety in cancer patients using the hospital anxiety and depression Scale. General Hospital Psychiatry 1993;15:69-74.

18. Chaturvedi S, Chandra K. Detection of anxiety and depression in cancer patients. NIMHANS J 1994;12:141-144.

19. Bjelland I, Dahl AA, Haugc TT, Neckelmannd D. The validity of the hospital anxiety and depression Scale An updated literature review. J Psychosomatic Research 2002;52:69-77.

20. Rico JL, Restrepo M, Molina M. Adaptación y validación de la escala hospitalaria de ansiedad y depresión (HAD) en una muestra de pacientes con cáncer del instituto nacional de cancerología de Colombia. Avances Medición 2005;3:73-86.

21. Alexander S, Palmer C, Stone PC. Evaluation of screening instruments for depression and anxiety in breast cancer survivors. Breast Cancer Res Treat 2010;122:573-578.

22. Özalp E, Soygür H, Cankurtaran E, Turhan L et al. Psychiatric morbidity and its screening in Turkish women with breast cancer: a comparison between the HADS and SCID tests. Psycho-Oncology 2008;17:668-675.

23. Villegas Pérez GC. Reporte de experiencia profesional. Tesis de maestría en Psicología. México: Facultad de Psicología, UNAM; 2004.

24. Rojas GC. Evaluar la validez de la escala de depresión geriátrica (GDS) y la escala de ansiedad y depresión para hospitales generales (HADS). Tesis de maestría. México: Fac de Medicina, Depto d Psiquiatría, UNAM; 1991.
25. Whaley JA. Diagnóstico de ansiedad y depresión en pacientes quemados (Escala HAD como una herramienta diagnóstica). Tesis de maestría. México: Fac de Medicina, Depto de Psiquiatría, UNAM; 1992.

26. López JC, Verónica V, Martínez A, Sierra AE et al. Exactitud y utilidad diagnóstica del hospital anxiety and depression Scale (HAD) en una muestra de sujetos obesos mexicanos. Rev Invest Clin 2002;54(5):403-409.

27. Beck A, Ward C, Mendelson M, Mock J, Erbaungh J. An inventory for measuring depression. Archives General Psychiatry 1961;4:53-63.

28. Jurado CS, Villegas E, Méndez SL, Rodríguez F et al. La estandarización del inventario de depresión de Beck para los residentes de la Ciudad de México. Salud Mental 1998;21(3)26-31.

29. Suarez GVC. Validación de la Escala de automedición de depresión de Zung en adolescentes mexicanos de secundaria mexicanos. Tesis de licenciatura. México: Facultad de Piscología, UNAM; 1988.

30. Beck AT, Epstein N, Brown G, Steer RA. An inventory for measuring clinical anxiety: psychometric properties. J Consulting Clinical Psychology 1988;56:893-897.

31. Robles R, Varela R, Jurado S, Páez F. Versión mexicana del inventario de ansiedad de Beck: Propiedades psicométricas. Revista Mexicana Psicología 2011;8:211-217.

32. Holland JC. NCCN Practice guidelines for the management of psychosocial distress. Oncology 1999;13(15A):113-147.

33. Almanza JJ, Rosario I, Pérez S. Traducción, adaptación y validación del termómetro de distréss en una muestra de pacientes mexicanos con cáncer. Rev Sanid Milit Mex 2008;62(5):209-217.

34. Vázquez OG, Martínez MM, Ugalde SC, Castillo ER et al. Tamizaje de aspectos psico-oncológicos: validación de una lista de chequeo. Psicooncología 2013;10(2-3):407-415.

35. Sellick SM, Edwardson AD. Screening new cancer patients for psychological distress using the hospital anxiety and depression scale. Psycho-Oncology 2007;16:534-542.

36. Tatrow K, Montgomery GH. Cognitive behavioral therapy techniques for distress and pain in breast cancer patients: A Meta-analysis. J Behavioral Medicine 2010;29(1):17-27.

37. Penedo FJ, Traeger L, Dahn J, Molton I et al. Cognitive behavioral stress management intervention improves quality of life in Spanish monolingual hispanic men treated for localized prostate cancer: Results of a randomized controlled trial. International J Behavioral Medicine 2007;14(3):164-172. 